\title{
Soins virtuels innovants pour la ventilation mécanique à domicile
}

\author{
Reshma Amin MD, Regina Pizzuti TRA BA, Francine Buchanan MBSI, Louise Rose PhD MSc inf
}

\author{
E Citation : CMAJ 2021 April 26;193:E607-11. doi : 10.1503/cmaj.202584-f
}

Voir la version anglaise de l'article ici : www.cmaj.ca/lookup/doi/10.1503/cmaj.202584

$\mathbf{L}$ e programme LIVE (Long-Term In-Home Ventilator Engagement) est un nouveau programme de soins virtuels, qui a été déployé pendant la pandémie de maladie à coronavirus 2019 (COVID-19) pour permettre aux centres spécialisés d'offrir un soutien virtuel aux personnes qui ont recours à la ventilation mécanique à domicile (VMD). À l'heure actuelle, 251 patients inscrits à 8 programmes de VMD hospitaliers ontariens (pédiatriques et adultes) participent au programme LIVE.

La ventilation mécanique à domicile est l'un des traitements à long terme hautement spécialisés et complexes les plus avancés offerts aux patients à l'extérieur de l'hôpital. Lors de travaux antérieurs menés à partir de bases de données médicoadministratives, notre groupe avait estimé la prévalence de la VMD au Canada à 12,9/100 000 habitants $^{1}$. Ces patients sont de fréquents utilisateurs du système de santé, ce qui entraîne des coûts élevés. Dans les 12 mois suivant l'instauration de la VMD, $49,9 \%$ des patients se présentent aux urgences, $29,9 \%$ sont hospitalisés et $43,9 \%$ utilisent des services de santé à domicile; seulement 75,3\% d'entre eux survivent $1 \mathrm{an}^{2}$. Les coûts mensuels médians en soins de santé publics et privés sont estimés à 8733 \$ (écart interquartile [EI] 5868 \$-15274\$) pour les patients sous ventilation effractive, et à 3925 (El 1212 \$-7390\$) pour les patients à domicile sous ventilation non effractive ${ }^{3}$.

La pandémie de COVID-19 a fait peser une menace sans précédent sur le système de santé canadien ${ }^{4}$. Les utilisateurs de ventilation mécanique à domicile sont exposés à un risque accru d'aggravation de leur insuffisance respiratoire et de décès secondaires à une infection par le coronavirus du syndrome respiratoire aigu sévère 2 (SRAS-CoV-2), en raison de leur insuffisance respiratoire chronique ${ }^{5,6}$. Hospitaliser les utilisateurs de VMD mobilise des appareils dont les hôpitaux ont cruellement besoin pour d'autres patients et pourrait mener à la prise de décisions très délicates quant à ceux qui auront accès à la ventilation ${ }^{7}$. Les patients sous VMD craignent particulièrement l'exposition au SRAS-CoV-2 et tendent à éviter les déplacements dans la collectivité, y compris pour leurs consultations de suivi à l'hôpital. La télémédecine est une solution qui permet d'offrir des soins centrés sur les patients et leurs proches, facilite l'échange de connaissances entre les divers intervenants et assure un accès dans des délais raisonnables aux médecins ayant l'expertise requise, tout en abolissant les contraintes de distance, de coût et de temps ${ }^{8}$. La pandémie de COVID-19 a illustré la

\section{POINTS CLÉS}

- Le programme ontarien LIVE (Long-Term In-Home Ventilator Engagement) propose une intervention de télémédecine à plusieurs volets comprenant des consultations virtuelles à domicile sécurisées, des plans de soins adaptables et des protocoles cliniques en fonction des signes et symptômes respiratoires, de même que des réponses aux problèmes de ventilateur, la télésurveillance des oxymètres et des ventilateurs et une bibliothèque de ressources intégrée.

- À ce jour, le programme LIVE est venu en aide à 251 enfants et adultes sous ventilation mécanique à domicile inscrits à 8 programmes ontariens.

- Les patients et les familles font état de sentiments de « connexion, d'autonomie et de sécurité » et les professionnels de la santé mentionnent « une relation facile en plus des bienfaits du programme pour la résolution des problèmes de ventilateur et l'amélioration des soins cliniques. »

nécessité de mettre sur pied un programme de télémédecine innovant pour les utilisateurs de VMD afin de préserver la santé de ces patients et de leur éviter de sortir de chez eux.

\section{En quoi consiste le programme LIVE (Long-Term In-Home Ventilator Engagement)?}

Le programme LIVE est un programme de prise en charge clinique intensive administré au moyen d'une plateforme de télémédecine (aTouchAway d'Aetonix), qui forme un réseau de soins composé d'équipes cliniques de VMD, de spécialistes du soutien technologique pour les ventilateurs (Ontario Ventilator Equipment Pool [ON-VEP]), du patient et de ses aidants. Le programme LIVE a été conçu conjointement par des patients, des familles, des professionnels de la santé et des chercheurs cliniciens, en collaboration avec notre partenaire de télémédecine. En amenant l'expertise des spécialistes de la VMD virtuellement au domicile des patients, le programme LIVE abolit les obstacles liés à la distance et aux déplacements pour ces patients souvent fragiles et dépendant d'un équipement complexe et difficile à transporter, en plus de réduire leur risque d'exposition au SRASCoV-2. Avant la pandémie de COVID-19, la norme de soins comprenait des consultations en personne avec l'équipe spécialisée 
en VMD tous les 3 à 6 mois pour une évaluation clinique et le téléchargement des données du ventilateur. De plus, les inhalothérapeutes de l'équipe ON-VEP sont disponibles en tout temps pour les questions liées à l'équipement. Le programme LIVE comporte une intervention en plusieurs volets qui comprend des consultations virtuelles à domicile, des plans de soins adaptables et des protocoles cliniques pour les divers signes et symp- tômes respiratoires, en plus d'aider à régler les problèmes de ventilateur. La plateforme permet la télésurveillance des oxymètres et des ventilateurs, et assure une communication sécurisée par messages texte, audio et vidéo entre les patients, leurs proches et l'équipe soignante (tableau 1 , figure 1 et figure 2). Même si d'autres programmes de télésurveillance ont été mis sur pied et évalués ${ }^{9}$, à notre connaissance, il n'existe actuellement

Tableau 1 : Composantes virtuelles du programme LIVE 9

\section{Composante}

Télémédecine

Télécommunications

Téléconsultation

Télésurveillance

Télétraitement

Téléconférence

Encadrement à

distance

\section{Description}

Les membres des équipes de VMD et du réseau ON-VEP utilisent la messagerie sécurisée, les protocoles et le téléchargement des données des ventilateurs pour offrir une prise en charge clinique

Communication bidirectionnelle sécurisée par messages texte, audio ou vidéo entre les patients, les proches et l'équipe soignante Consultation ponctuelle initiée par les patients et leurs proches et fournie par les membres des équipes VMD et du réseau ON-VEP pour la résolution des symptômes cliniques ou des problèmes de ventilateurs

Connectivité cellulaire et technologie Bluetooth pour le téléchargement des données des ventilateurs

Les consultations ponctuelles et l'interprétation des données téléchargées concernant les respirateurs peuvent mener à des modifications de leurs réglages

Communications audio et vidéo tridirectionnelles, qui peuvent être initiées par les patients, les proches ou l'équipe soignante

Des conseils et de l'aide à la résolution de problèmes peuvent être fournis par un membre de l'équipe de VMD si les données téléchargées des ventilateurs révèlent une piètre observance thérapeutique

Remarque : LIVE = Long-term In-Home Ventilator Engagement, ON-VEP = Ontario Ventilator Equipment Pool, VMD = ventilation mécanique à domicile.

Un système de communication bidirectionnelle permet aux patients, aux familles ou à l'équipe de VMD d'engager une conversation texte, audio ou vidéo.

\begin{abstract}
Les consultations cliniques virtuelles périodiques se passent comme les visites en personne: le médecin vérifie comment le patient utilise la technologie respiratoire et les réglages du ventilateur, en plus de répondre à ses questions ou préoccupations à ce sujet.

Questionnaires de surveillance dynamique tous les mois ou les deux mois. Les questionnaires permettent de suivre les symptômes du patient et les problèmes de ventilateur. Un code de couleur vert, jaune ou rouge - est assigné au patient selon ses réponses, qui sont revues par l'équipe soignante et abordées avec la famille.
\end{abstract}

Une surveillance à distance du ventilateur est assurée en temps réel par téléchargement via la technologie Bluetooth ou un appareil cellulaire pour évaluer l'observance, les fuites et l'efficacité.
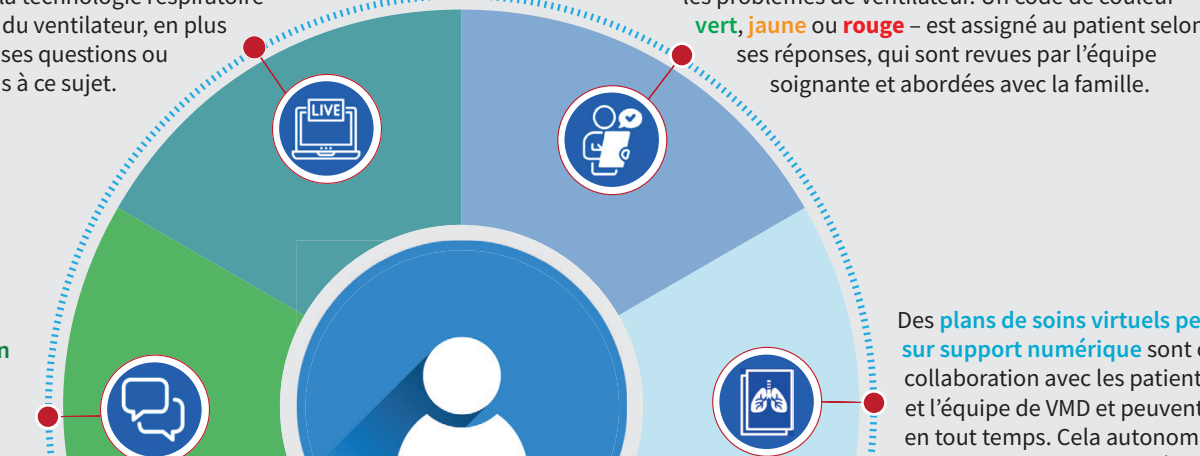

Des plans de soins virtuels personnalisés sur support numérique sont conçus en collaboration avec les patients, les familles et l'équipe de VMD et peuvent être mis à jour en tout temps. Cela autonomise les patients et leur donne la possibilité de voir et de demander des changements à leur plan de soins, ce qui améliore leur sécurité.

Figure 1 : Les 6 principes du programme LIVE (Long-Term In-Home Ventilator Engagement). 
aucune autre intervention de télémédecine similaire pour les utilisateurs de VMD au Canada ou ailleurs dans le monde.

Lorsque l'incidence des infections par le SRAS-CoV-2 a commencé à augmenter au Canada, nous étions à développer l'intervention virtuelle LIVE en vue d'un essai randomisé et contrôlé subventionné par les Instituts de recherche en santé du Canada. La pandémie a accéléré le déploiement clinique de l'intervention dans 8 programmes de VMD.

\section{Comment le programme LIVE est-il exécuté et qui y est admissible?}

Les équipes spécialisées en VMD repèrent les patients exposés à un risque élevé d'exacerbation de leur maladie respiratoire (p. ex., patients sous ventilation effractive et dont la maladie sous-jacente évolue rapidement ou patients médicalement fragiles). Avec le soutien de l'équipe ON-VEP, l'application du programme est téléchargée sur l'appareil du patient (ou d'un parent ou d'un aidant). Si les patients n'ont pas d'appareil compatible avec l'application ou si leur accès Internet est inadéquat, nous leur prêtons une tablette munie d'une carte SIM (subscriber identification module). Le patient est alors accueilli et orienté dans le programme LIVE. Un membre de l'équipe prend contact avec lui par l'application pour lui expliquer les fonctionnalités et les composantes du programme, et pour l'aider à préparer le plan de soins virtuel avec ses aidants. Une fois cette étape franchie, le patient apparaît sur le tableau de bord de l'équipe de VMD, et la communication clinique bidirectionnelle peut commencer. Pour l'instant, 251 patients de 8 programmes de VMD hospitaliers (enfants et adultes) ontariens participent au programme LIVE (figure 3).

\section{Quelles ont été les conséquences imprévues du déploiement rapide du programme LIVE?}

Quatre principaux problèmes sont survenus lors du déploiement rapide du programme LIVE. Premièrement, les patients sous VMD en Ontario n'avaient pas tous le même accès au programme LIVE. Seuls les 8 programmes recrutés en vue de l'essai randomisé et contrôlé connaissaient l'application et pouvaient participer. Deuxièmement, les exigences juridiques en matière de
A) Communication bidirectionnelle simple entre patients, familles et fournisseurs de soins.

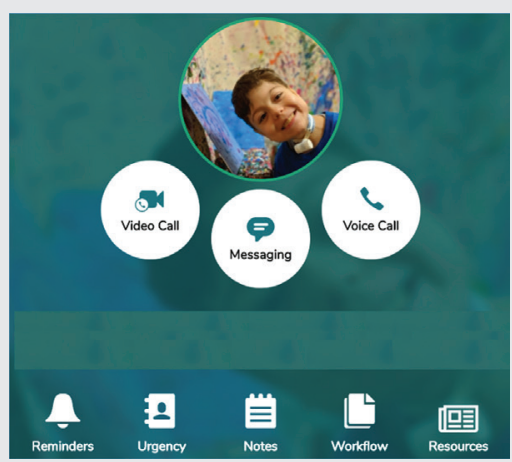

C) Surveillance intensive par des questionnaires et le téléchargement des données du ventilateur.
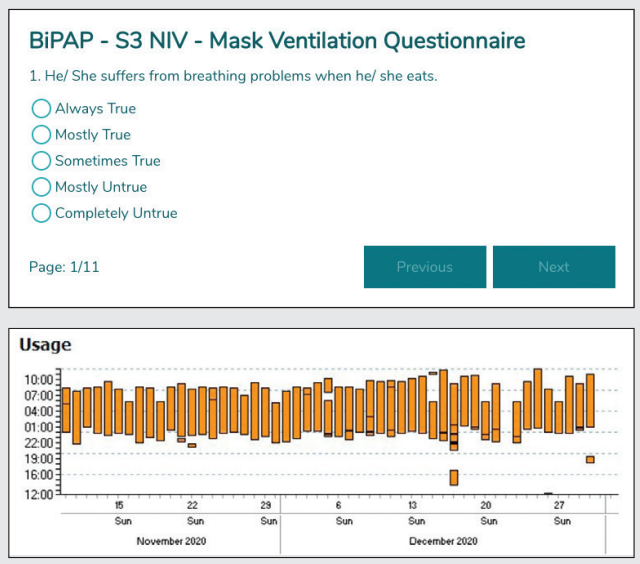

B) Plan de soins virtuels créé et mis à jour en collaboration avec les patients, la famille et les fournisseurs de soins.

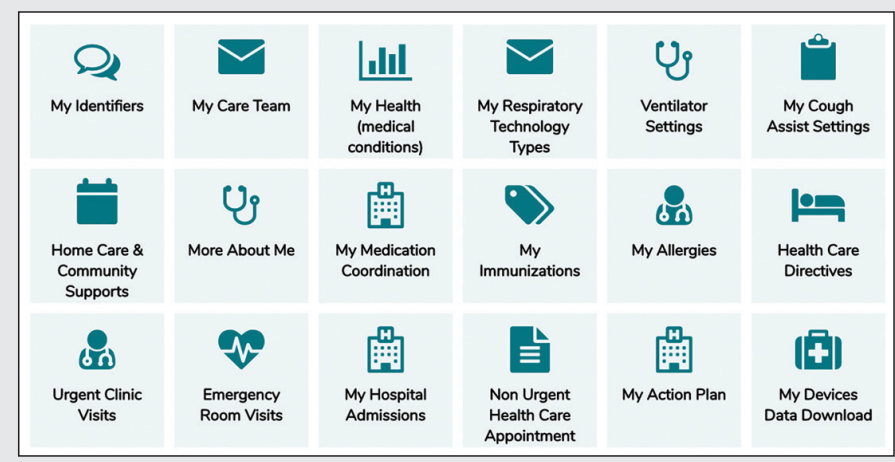

D) Bibliothèque de ressources éducatives, comprenant des documents et des vidéos offrant des directives pour l'utilisation, l'entretien et le dépannage des appareils.

\section{Oxygen saturation monitoring: Frequently Asked Questions}

\section{How does an oximeter work?}

During use, the oximeter continuously calculates and gives a reading of the oxygen saturation and the heart rate. The beep's pitch drops as oxygen saturation falls and rises as it recovers. This is an important safety feature, as it allows you to hear changes in your child's oxygen saturation immediately without needing to look at the monitor all the time.

The probe you place on your child has a small red light on one side and a detector on the other side. The red light shines through your child's finger or toe and is seen by the detector on the other side. The detector measures the amount of oxygen in the blood.

For the oximeter to function, the sensor must be placed where a pulse can be detected, such as on the finger, foot, toe or earlobe.

Figure 2: Captures d'écran (en anglais) montrant les caractéristiques du programme LIVE, reproduit avec l'autorisation d'Aetonix. 


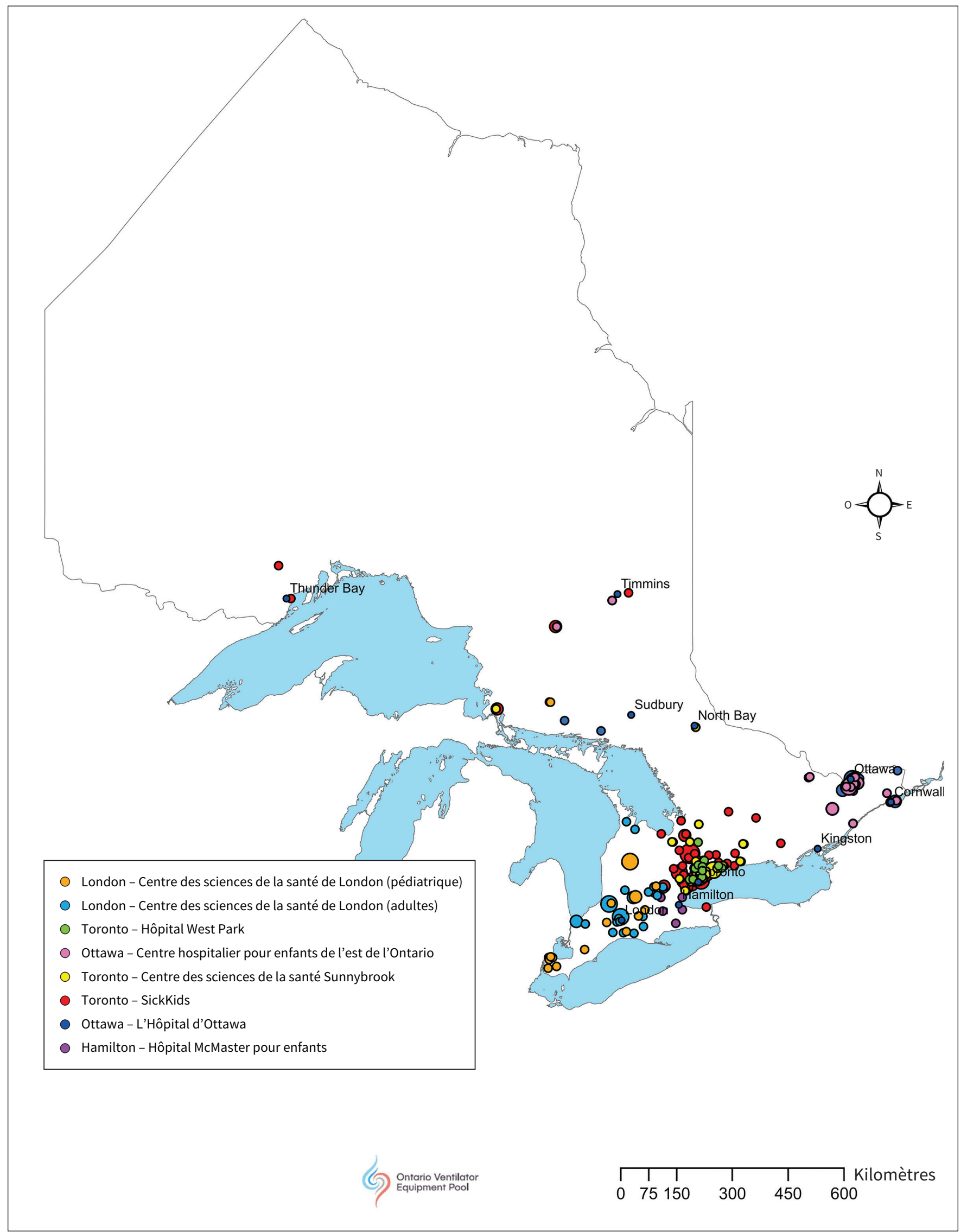

Figure 3 : Distribution géographique des 251 participants au programme LIVE par région de tri d'acheminement. Carte préparée et reproduite avec l'autorisation de Tom Fisher, TRA, le 16 février 2021. 
protection des renseignements personnels appliquées aux programmes de soins virtuels n'étaient pas uniformes entre les établissements, entraînant des retards dans l'établissement des contrats et limitant le nombre de patients inscrits dans certains centres. Troisièmement, en raison du déploiement rapide, il a été impossible d'intégrer les dossiers médicaux électroniques, d'où un certain dédoublement de documents. Quatrièmement, malgré nos efforts pour surmonter les inégalités en matière d'accès aux technologies numériques (tablettes et cartes SIM), nous n'avons pu inclure que les patients de langue anglaise ou française, en raison du manque d'interprètes pour les consultations virtuelles.

\section{À ce jour, quelles données le programme a-t-il générées?}

Dans d'autres contextes, les soins virtuels dispensés aux utilisateurs de VMD ont contribué à l'efficacité des précieuses ressources en santé en réduisant les consultations aux urgences et le coût des soins, pour les patients, leurs proches et le système de santé ${ }^{10-13}$. À ce jour, les patients et les proches aidants inscrits au programme LIVE affirment que ce dernier leur procure un sentiment « de connexion, d'autonomie et de sécurité ». Dans leurs commentaires, les équipes de VMD font état d'une relation facile avec les familles et d'une économie de temps en plus des bienfaits cliniques de la télésurveillance de la VMD. Le programme a aussi contribué à prévenir les consultations pour soins aigus, comme l'illustre le cas d'une jeune fille de 11 ans à qui on avait prescrit une ventilation non effractive. Elle a répondu à notre questionnaire hebdomadaire, faisant état de désaturations intermittentes en oxygène la nuit, même si elle se sentait bien. Les données du respirateur obtenues à distance ont révélé à l'équipe de VMD qu'il fallait régler à la hausse la ventilation alvéolaire cible. Ces changements ont été effectués par téléphone et, après leur mise en œuvre, la situation est rentrée dans l'ordre. Autrement, cette patiente aurait dû se rendre dans une clinique ou un service d'urgence pour y être prise en charge. Notre groupe compilera les données probantes officielles du programme LIVE à l'aide d'une évaluation à méthodes mixtes, incluant la faisabilité et l'acceptabilité.

\section{Que nous réserve l'avenir?}

La pandémie de COVID-19 a changé le paysage de la médecine clinique et la prestation des services de santé. Selon nous, les soins virtuels sont appelés à faire partie intégrante de la trousse d'outils cliniques pour la population sous VMD. Les leçons tirées du déploiement clinique du programme LIVE et de notre essai randomisé et contrôlé (recrutement commencé au début 2021) peuvent être mises à profit pour la mise à l'échelle et l'évaluation nationale des soins virtuels à l'intention des patients sous VMD. De plus, il sera possible de promouvoir son intégration avec le dossier médical électronique et d'appliquer l'expérience provinciale du programme LIVE à d'autres populations de patients vulnérables aux besoins complexes vivant dans la collectivité et dans des établissements de soins de longue durée.

\section{Références}

1. Rose L, McKim DA, Katz SL, et al. Home mechanical ventilation in Canada: a national survey. Respir Care 2015;60:695-704.

2. Povitz M, Rose L, Shariff SZ, et al. Home mechanical ventilation: a 12-year populationbased retrospective cohort study. Respir Care 2018;63:380-7.

3. Nonoyama ML, McKim DA, Road J, et al. Healthcare utilisation and costs of home mechanical ventilation. Thorax 2018 Jan. 26 [cyberpublication avant impression]. doi: 10.1136/thoraxjnl-2017-211138.

4. Shoukat A, Wells CR, Langley JM, et al. Projecting demand for critical care beds during COVID-19 outbreaks in Canada. CMAJ 2020;192:E489-96.

5. Sanchez-Ramirez DC, Mackey D. Underlying respiratory diseases, specifically COPD, and smoking are associated with severe COVID-19 outcomes: a systematic review and meta-analysis. Respir Med 2020;171:106096.

6. Sanyaolu A, Okorie C, Marinkovic A, et al. Comorbidity and its impact on patients with COVID-19. SN Compr Clin Med 2020 June 25 [cyberpublication avant impression]. 1-8. doi: 10.1007/s42399-020-00363-4.

7. Barrett K, Khan YA, Mac S, et al. Estimation of COVID-19-induced depletion of hospital resources in Ontario, Canada. CMAJ 2020;192:E640-6.

8. Jamieson T, Wallace R, Armstrong K, et al. Virtual care: a framework for a patientcentric system. Toronto: Women's College Hospital; 2015. Accessible ici : www. womenscollegehospital.ca/assets/pdf/wihv/WIHV_VirtualHealthSymposium.pdf (consulté le 15 févr. 2021).

9. Ambrosino N, Vitacca M, Dreher M, et al.; ERS Tele-Monitoring of VentilatorDependent Patients Task Force. Tele-monitoring of ventilator-dependent patients: a European Respiratory Society Statement. Eur Respir J 2016;48:648-63.

10. Muñoz-Bonet JI, López-Prats JL, Flor-Macián EM, et al. Usefulness of telemedicine for home ventilator-dependent children. J Telemed Telecare 2020;26:207-15.

11. Lopes de Almeida JP, Pinto AC, Pereira J, et al. Implementation of a wireless device for real-time telemedical assistance of home-ventilated amyotrophic lateral sclerosis patients: a feasibility study. Telemed J E Health 2010;16:883-8.

12. Bertini S, Picariello M, Gorini M, et al. Telemonitoring in chronic ventilatory failure: a new model of survellaince, a pilot study. Monaldi Arch Chest Dis 2012;77:57-66.

13. Vitacca M, Bianchi L, Guerra A, et al. Tele-assistance in chronic respiratory failure patients: a randomised clinical trial. Eur Respir J 2009;33:411-8.

\section{Intérêts concurrents : Aucun déclaré.}

Cet article a été révisé par des pairs.

Les auteurs ont obtenu le consentement des patients.

Affiliations : Division de pneumologie (Amin), Département de pédiatrie, Hôpital pour enfants malades de Toronto (SickKids); Institut de recherche du SickKids (Amin, Buchanan), Toronto, Ont.; The Ontario Ventilator Equipment Pool (Pizzuti), Centre des sciences de la santé de Kingston, Kingston, Ont.; Florence Nightingale Faculty of Nursing, Midwifery and Palliative Care (Rose), King's College, Londres, R.-U.

Collaborateurs : Tous les auteurs ont substantiellement contribué à la conception et à l'élaboration de ce projet. Reshma Amin a rédigé le manuscrit initial, que tous les auteurs ont révisé de manière critique pour le contenu intellectuel. Les auteurs ont tous approuvé la version finale destinée à être publiée et assument l'entière responsabilité de tous les aspects du travail.

Propriété intellectuelle du contenu : Il s'agit d'un article en libre accès distribué conformément aux modalités de la licence Creative Commons Attribution (CC BY-NC-ND 4,0), qui permet l'utilisation, la diffusion et la reproduction dans tout médium à la condition que la publication originale soit adéquatement citée, que l'utilisation se fasse à des fins non commerciales (c.-à-d., recherche ou éducation) et qu'aucune modification ni adaptation n'y soit apportée. Voir : https://creativecommons.org/licenses/by-nc-nd/4.0/deed.fr.

Financement : Ce projet a bénéficié du soutien du réseau Ontario Ventilator Equipment Pool, exploité par le Centre des sciences de la santé de Kingston et subventionné par le Programme d'appareils et accessoires fonctionnels du ministère ontarien de la Santé.

Correspondance : Reshma Amin, reshma.amin@sickkids.ca 